El estudio del lavado broncoalveolar era un frotis inflamatorio agudo, siendo negativo para células malignas y micobacterias. Se realizó toracotomía y biopsia de una adenopatía mediastínica que mostró una adenitis reactiva con una moderada antracosis sin invasión neoplásica.

En la ecografía abdominal se observaba una hepatomegalia homogénea, con vía biliar intrahepática izquierda discretamente dilatada, un colédoco de 6’3 cm

Con el diagnóstico de neumonía por $R$. typhi se inició tratamiento con doxiciclina $100 \mathrm{mg} / 12$ horas, observándose una buena evolución clínica y radiológica. En la TAC torácica de control solamente se observaron unos tractos lineales fibróticoresiduales en el LSD.

El tifus murino es causado por la Rickettsia typhi, está infección está infradiagnosticada, se considera que tiene un curso leve, pudiendo llegar a ocasionar epidemias, su prevalencia es alta en determinadas regiones. Se transmite al ser humano cuando la pulga de la rata infectada realiza una picadura en la piel del huésped y deposita en la herida las heces contaminadas, la distribución es mundial. La $R$. typhi puede afectar a todos los órganos, produciendo una vasculitis linfohistiocítica en las que se puede identificar al germen; se puede manifestar como neumonitis, nefritis, miocarditis, hepatitis, etc. En la mayor parte de casos no se suele recordar la picadura, y solo aparecen síntomas inespecíficos como fiebre, cefalea, y mialgias. La erupción cutánea aparece entre un 20-50\% de los pacientes, a veces la erupción es de pequeño tamaño, desapareciendo posteriormente en 4 a 8 días, esto hace que la identificación de la afectación cutánea sea difícil y que no se pueda descartar la enfermedad en los pacientes que no se ha diagnosticado. El curso clínico suele ser benigno, apareciendo en ocasiones síntomas respiratorios hasta en un $35 \%$ de pacientes, afectación del sistema nervioso central, hemorragia digestiva, insuficiencia renal, e insuficiencia hepática; en raras ocasiones la evolución puede ser fatal. La afectación hepática produce alteraciones bioquímicas como son moderada elevación de la AST, ALT, fosfatasa alcalina y LDH, aunque en este caso las alteraciones se atribuyeron a la hepatopatía crónica que padecía el paciente.

El diagnóstico de la enfermedad se basa en la sospecha clínica, y la realización de pruebas serológicas, siendo los más útiles aquellos que emplean antígenos específicos de $R$. typhi, como la inmunofluorescencia indirecta, con la que se pueden detectar títulos de diagnósticos de anticuerpos a los 10 días del inicio de la enfermedad. Con este método se pueden detectar títulos elevados de anticuerpos en un $50 \%$ de los pacientes en la primera semana y casi en el $100 \%$ a los 5 días de haberse iniciado la enfermedad, hay que tener en cuenta la aparición de reacciones cruzadas con la R conorii. En el caso expuesto el diagnóstico fue serológico al comprobar un aumento en el título de anticuerpos, y que el título de anticuerpos fue muy superior el caso de la $R$. typhi.

Se descartaron otras causas infecciosas y neoplásicas que pudieran ocasionar la sintomatología del paciente presentado. En el caso presentado el paciente no era inmigrante, y tampoco había realizado ningún viaje al extranjero, pero probablemente las condiciones higiénicas en las que vivía el paciente eran pésimas. Por todo lo expuesto anteriormente habría que tener en cuenta el diagnóstico de esta enfermedad en los casos de fiebre prolongada en España.

Una vez realizado el diagnóstico se inició tratamiento con doxiciclina evidenciándose mejoría del estado general con disminución del tamaño de los infiltrados pulmonares y de las adenopatías mediastínicas.

F. J. Nicolás Sánchez, I. Ribes Amorós, M. Cervera del Pino, R. M. Sarrat Nuevo, J. Bestard Solivellas, J. Cabau Rubies

Servicio de Medicina Interna. Hospital de Santa María. Lleida
1. Botelho-Nevers E, Raoult D. Fever of unknown origin due to rickettsioses. Infect Dis Clin North Am 2007; 21 (4): 997-1011.

2. Basrai D, Pox C, Schmiegel W. Fever of intermediate duration after return from the Canary Islands. Internist (Berl) 2007; 48: 413-9.

3. Letaïef AO, Kaabia N, Chakroun M, Khalifa M, Bouzouaia N, Jemni L. Clinical and laboratory features of murine typhus in central Tunisia: a report of seven cases. Int J Infect Dis 2005; 9: 331-4. Epub 2005 Jul 27.

4. Murdoch DR, Woods CW, Zimmerman MD, Dull PM, Belbase RH, Keenan AJ, et al. The etiology of febrile illness in adults presenting to Patan hospital in Kathmandu, Nepal. Am J Trop Med Hyg 2004; 70 (6): 670-5.

5. Ruiz Beltrán R, Herrero Herrero JI, Martín Sánchez A, Martín González JA. Prevalence of antibodies to Rickettsia conorii, Coxiella burnetii an Rickettsia typhi in Salamanca province (Spain): serosurvey in the human population. Eur J Epidemiol 1990; 6: 293-9.

\section{Necrosis epidérmica tóxica por colirio de dorzolamida}

\section{Sr. Director:}

La necrólisis epidérmica tóxica (NET) es una enfermedad cutánea que pone en peligro la vida y se caracteriza por la formación de ampollas y la exfoliación de la piel. Puede ser provocada por la reacción a un fármaco, frecuentemente antibióticos o anticonvulsionantes aunque aproximadamente en un tercio de los casos de necrólisis epidérmica tóxica, no es posible identificar la causa (1). El clorhidrato de dorzolamida al $2 \%$ es un potente inhibidor de las isoenzimas II y IV de la anhidrasa carbónica humana (IAC), con buena penetración intraocular después de la administración tópica, reduciendo la presión intraocular como consecuencia de una disminución del flujo de entrada del humor acuoso por acción local. El perfil de seguridad de la dorzolamida es bueno, y así se confirma en más del millón de paciente que lo utilizan satisfactoriamente (2). Sólo se han publicado dos artículos de NET por colirio de dorzolamida (3-4). En este trabajo se presenta el caso de una reacción adversa sistémica grave a la dorzolamida que ha sido tratada en nuestro servicio.

Paciente de 72 años sin antecedentes de interés y sin tratamiento farmacológico previo que fue tratada por un glaucoma de ángulo abierto con dorzolamida $\left(\right.$ Trusopt $\left.^{\circledR}\right)$ tres veces al día durante 8 días y que fue hospitalizada por una erupción ampollosa que afectaba a la mucosa nasal, oral y ocular e interesaba al $40 \%$ de la superficie cutánea con despegamiento de amplias zonas epidérmicas que dejaban erosiones exudativas y que fue diagnosticado de necrólisis epidérmica tóxica. Excepto el balance hidroelectrolítico, los parámetros renales, hematológicos y la función hepática estaban dentro de los límites normales. Se suspendió la dorzolamida y la paciente fue tratada con inyecciones de maleato de feniramina y prednisolona $1 \mathrm{~g}$ intravenoso durante 4 días. La mucosa oral se trató con metronidazole $1 \%$ y lavados con clorhexidina. Al quinto día se comenzó con prednisolona oral $40 \mathrm{mg}$ hasta el día 20 , todo con monitorización en un área de cuidados intensivos. Durante este periodo la paciente mejoró gradualmente pero con secuales oftalmológicas muy graves (Fig 1).

Cada ml de solución oftálmica de Trusopt contiene $20 \mathrm{mg}$ de dorzolamida (22.3 mg de dorzolamida hidroclorhidrato). La absorción sistémica es a través de la conjuntiva y por la muco- 


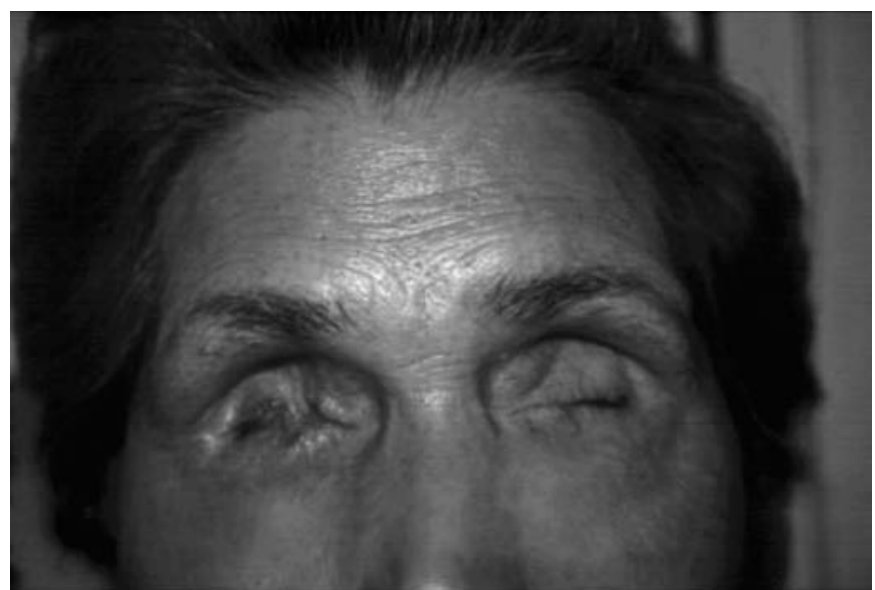

Fig. 1. Pérdida de la estructura ocular bilateral con sinequias palpebrales y conjuntivales extensas.

sa nasal. Dorzolamida en la circulación sistémica se une a las proteínas plasmáticas en un $33 \%$ y se excreta sin cambios a través de la orina junto a su metabolito. La NTE es la reacción cutánea más grave ocasionada por la ingesta de medicamentos, se caracteriza por exfoliación epidérmica, mucositis y compromiso sistémico que traen consigo altas cifras de morbimortalid (1). Un gran número de fármacos pueden precipitarla, los agentes etiológicos más frecuentes son la fenitoína y las sulfonamidas (1). La suspensión precoz del fármaco responsable se asocia con un mejor pronóstico y con una menor mortalidad. Los inhibidores de la anhidrasa carbónica son fármacos cuya estructura molecular tiene en común la unión de un grupo sulfonamídico (-SO2NH2) no sustituído a un anillo aromático (5). A diferencia de los inhibidores de la anhidrasa carbónica (IAC) por vía oral, Trusopt ${ }^{\circledR}$ se aplica tópicamente y ejerce sus efectos directamente en el ojo (2). Comparado con los IAC orales la aplicación tópica produce una baja incidencia de efectos sistémicos secundarios. A nuestro criterio, este caso es interesante por ser clínicamente relevante al poner en peligro la vida del paciente y por las graves secuelas oftalmológicas, porque casi no hay referencia en la bibliografía de NET y fármacos de aplicación tópica oftálmica, y para llamar la atención en la utilización de estos fármacos en pacientes "suceptibles"

\section{V.M. Asensio-Sánchez}

Hospital General. Servicio Castellano-Leonés de Salud. Servicio de Oftalmología. Medicina del Campo. Valladolid

1. García-Doval I, Roujeau J, Cruces M. Necrólisis epidérmica tóxica y síndrome de Stevens-Johnson: Clasificación y actualidad terapéutica. Actas Dermosifiliogr 2000; 91: 541-51.

2. Balfour JA, Wilde MI. Dorzolamide. A review of its pharmacology and therapeutic potential in the management of glaucoma and ocular hypertension. Drug Aging 1997; 10: 384-403.

3. Schmutz JL, Barbaud A, Trechot P. Toxic epidermal necrolysis following eyedrop treatment for glaucoma. Ann Dermatol Venereol 2007; 134: 417

4. Florez A, Rosón E, Conde A, González B, García-Doval I, de la Torre $\mathrm{C}$, et al. Toxic epidermal necrolysis secondary to timolol, dorzolamide, and latanoprost eyedrops. J Am Acad Dermatol 2005; 53: 909-11.

5. Kumar S, Singh V, Tiwari M. Quantitative structure activity relationship studies of sulfamide derivatives as carbonic anhydrase inhibitor: As antiglaucoma agents. Med Chem 2007; 3: 379-86.
Ingresos en HADO desde el Servicio de Urgencias

\section{Sr. Director:}

La Hospitalización a Domicilio (HADO) es una modalidad asistencial, remonta su inicio al año 1947 cuando en el Hospital Universitario Guido Montefiore (Nueva York, EE.UU.) se crea la primera Unidad de Home Care ideada por el Dr. E.M. Bluestone como una "extensión del propio hospital hacia el domicilio del paciente. En 1951 el Dr. Siguier pone en marcha en el Hospital Tenon (Paris, Francia) la primera experiencia francesa y en 1975 se crea la primera estructura de Hospitalización a Domicilio en la Asistencia Pública para los hospitales de París. En España este tipo de asistencia se introduce en 1982 cuando el actual Hospital Gregorio Marañón (entonces H. Provincial de Madrid) implanta su primera Unidad creándose posteriormente en 1983 la Unidad del Hospital de Cruces. Actualmente en todo el territorio nacional 68 centros cuentan con estas unidades (el 10\% de la red), aunque con una distribución irregular por las distintas Comunidades Autónomas. En Galicia las Unidades de Hospitalización a Domicilio han sido una prioridad estratégica del Servizo Galego de Saude. Como se puede apreciar este tipo de actividad es relativamente reciente en nuestro país pero empieza a tener una amplia difusión y experiencia. Su crecimiento es progresivo debido a la gran demanda generada ya que los resultados obtenidos en base a estudios realizados, demuestran una mayor calidad asistencial, reducción de costes, disponibilidad de camas, reducción de estancias $(1,2)$. Frente a la Atención Domiciliaria, de competencia exclusiva de la Atención Primaria, y orientada hacia los cuidados a largo plazo, las medidas preventivas y la educación para la Salud, los pacientes atendidos en HADO requieren cuidados lo suficientemente intensos y/o complejos como para no poder ser asumidos por los profesionales del nivel asistencial primario. $\mathrm{La}$ derivación a estas unidades suele hacerse por tanto desde el nivel asistencial hospitalizado. Existe poca información sobre los pacientes ingresados en situación aguda (Servicios de Urgencias) a estas unidades ya que no eran éstos los Servicios prioritarios para la derivación de patología a domicilio pero la experiencia demuestra que progresivamente la demande desde la sala de urgencias se mantiene creciente. Realizamos una evaluación de los pacientes ingresados en HADO desde el Servicio de Urgencias en el Complexo Hospitalario Universitario de santiago de Compostela.

En nuestra Unidad realizamos un estudio retrospectivo de los pacientes ingresados desde el Servicio de Urgencias en HADO durante el período entre el 1 de enero de 2004 al 31 de diciembre de 2006 mediante la revisión sistemática de las historias clínicas de los enfermos. Se valoraron los datos epidemiológicos así como el tipo de patología ingresada.

Durante este período se ingresaron en HADO 1495 pacientes de los que 142 (9\%), procedían del Servicio de Urgencias. La distribución por años es de un $8 \%$ del total de ingresos en la unidad en 2004 a un $14 \%$ en 2006. Las características epidemiológicas de los pacientes se recogen en la tabla I.

La hospitalización a domicilio surge como una fórmula alternativa a la hospitalización clásica con el fin de mejorar la gestión de las camas hospitalarias, de conseguir una mayor integración entre el paciente, su familia y el personal sanitario, y con el fin de servir de puente entre la Atención primaria y la especializada, contribuyendo a una mayor comunicación entre niveles, todo esto a la vez que se le proporcionan al paciente en su domicilio a asistencia necesaria hasta el alta clínica definitiva

Los servicios de Hospitalización a Domicilio están preparados para poder atender todo tipo de patología hospitalaria aguda en fase de estabilización $(1,2)$. El acceso desde el Servicio de Urgencias parece decantarse hacia pacientes crónicos en general con enfermedad compleja e intención paliativa para lo que esta 